OPEN ACCESS

Edited by:

Massimiliano de Zambotti, SRI International, United States

Reviewed by:

Toru Nakamura, Osaka University, Japan Merel Van Gilst,

Eindhoven University of Technology, Netherlands

${ }^{*}$ Correspondence:

Zilu Liang

liang.zilu@kuas.ac.jp

Specialty section This article was submitted to Health Informatics,

a section of the journal

Frontiers in Digital Health

Received: 09 February 2021 Accepted: 19 April 2021 Published: 28 May 2021

Citation: Liang Z and Chapa-Martell MA (2021) A Multi-Level Classification Approach for Sleep Stage Prediction With Processed Data Derived From Consumer Wearable Activity Trackers. Front. Digit. Health 3:665946. doi: 10.3389/fdgth.2021.665946

\section{A Multi-Level Classification Approach for Sleep Stage Prediction With Processed Data Derived From Consumer Wearable Activity Trackers}

\author{
Zilu Liang ${ }^{1,2 *}$ and Mario Alberto Chapa-Martell ${ }^{3}$ \\ 1 Ubiquitous and Personal Computing Laboratory, Faculty of Engineering, Kyoto University of Advanced Science, Kyoto, \\ Japan, ${ }^{2}$ Institute of Industrial Science, The University of Tokyo, Tokyo, Japan, ${ }^{3}$ Silver Egg Technology, Osaka, Japan
}

Consumer wearable activity trackers, such as Fitbit are widely used in ubiquitous and longitudinal sleep monitoring in free-living environments. However, these devices are known to be inaccurate for measuring sleep stages. In this study, we develop and validate a novel approach that leverages the processed data readily available from consumer activity trackers (i.e., steps, heart rate, and sleep metrics) to predict sleep stages. The proposed approach adopts a selective correction strategy and consists of two levels of classifiers. The level-I classifier judges whether a Fitbit labeled sleep epoch is misclassified, and the level-II classifier re-classifies misclassified epochs into one of the four sleep stages (i.e., light sleep, deep sleep, REM sleep, and wakefulness). Best epoch-wise performance was achieved when support vector machine and gradient boosting decision tree (XGBoost) with up sampling were used, respectively at the level-I and level-II classification. The model achieved an overall per-epoch accuracy of 0.731 \pm 0.119 , Cohen's Kappa of $0.433 \pm 0.212$, and multi-class Matthew's correlation coefficient (MMCC) of $0.451 \pm 0.214$. Regarding the total duration of individual sleep stage, the mean normalized absolute bias (MAB) of this model was 0.469 , which is a 23.9\% reduction against the proprietary Fitbit algorithm. The model that combines support vector machine and XGBoost with down sampling achieved sub-optimal per-epoch accuracy of $0.704 \pm 0.097$, Cohen's Kappa of $0.427 \pm 0.178$, and MMCC of $0.439 \pm 0.180$. The sub-optimal model obtained a MAB of 0.179 , a significantly reduction of $71.0 \%$ compared to the proprietary Fitbit algorithm. We highlight the challenges in machine learning based sleep stage prediction with consumer wearables, and suggest directions for future research.

Keywords: sleep tracking, machine learning, consumer sleep technology, wearable sleep trackers, Fitbit, ambulatory sleep monitoring, ubiquitous computing

\section{INTRODUCTION}

Measuring sleep over a prolonged period of time is important for understanding the day-to-day variability in sleep dynamics. Traditional sleep monitoring methods, such as polysomnography (PSG) and videosomnography are expensive, burdensome, and not suited for longitudinal sleep tracking. In contrast, ubiquitous sleep tracking 
technologies enable unobtrusive measurement of sleep over extended periods in free-living environments. A variety of ubiquitous sleep tracking systems have been developed, including bed sensors $(1,2)$, wireless EEG $(3,4)$, smartphone apps $(5,6)$, and wearable activity trackers (7). Activity trackers are among the most popular consumer sleep tracking technologies. The opportunities and challenges pertaining the personal use of these devices have been well-studied (8-12). In the meantime, recent advances in wearable technology has seen a rise in employing consumer activity trackers, such as Fitbit in research studies for measuring sleep outcomes (13-16). Some studies even leverage Fitbit sleep data as the ground truth to validate new sleep tracking devices (17). The popularity of activity trackers in the research community is mostly due to their appealing features: they are more affordable than medical sleep monitors and easy to use for longitudinal sleep data collection without the necessity of frequent technical support; they have well-designed dashboard to visualize sleep data and offer raw data retrieval at reasonably high granularity through Application Program Interface (API). All these features allure researchers to use these consumer devices as an alternative of medical sleep monitors, especially when research budget is limited and when sleep outcomes are not the main concern.

Nonetheless, there is strong evidence that consumer activity trackers have limited accuracy for measuring sleep (18-25). Previous validation studies show that many devices, such as Fitbit Flex and Fitbit HR Charge tend to overestimate sleep time while underestimate wake time compared to PSG $(22,26)$. The result was consistent for adolescents (21) and adults $(19,22)$. Recent validation studies of the latest Fitbit models (e.g., Fitbit Charge 2/3) further revealed that while new models could measure total sleep time and sleep efficiency with satisfactory accuracy, their capability in detecting sleep stages-wakefulness, light sleep, deep sleep and REM sleep-remains to be limited $(18,19)$. The disparity between Fitbit and medical devices may be more pronounced in free-living environments $(18,27)$ and among people with sleep problems (28). Furthermore, the accuracy of Fitbit demonstrates temporary patterns, with better accuracy for deep sleep in the first half of a night and better accuracy for REM sleep in the second half of a night (29).

The limitation in measurement accuracy of consumer activity trackers demands the development of new sleep staging algorithms. There is a large body of research on sleep staging with PSG signals, but these algorithms are not readily applicable to consumer activity trackers due to the difference in sensing modalities. A PSG test often involves the measurement of multiple modalities of physiological signals including electroencephalogram (EEG), electrooculogram (EOG), electromyogram (EMG), and electrocardiogram (ECG). In contrast, Fitbit devices only rely on two sensing modalitiesaccelerometer and PPG-and use these limited signals as input for sleep staging. Although these signals demonstrate sleep stage wise characteristics (30), they are insufficient for achieving satisfactory accuracy in classifying sleep stages (28).

In this study we propose a two-level classification approach for sleep staging with consumer activity trackers. The goal was to train a computational model that captures a mapping relation between the data derived from Fitbit and standard questionnaire to medical-grade sleep staging. To be more specific, the levelI classifier of the model judges whether a Fitbit labeled sleep stage epoch is misclassified, and the level-II classifier reclassifies misclassified epochs. The rationale behind the two-level approach is that a portion of Fitbit labeled sleep stage epochs is correct, and only incorrect labels need to be re-classified. In this study we select Fitbit as a representative of consumer activity trackers because of its popularity, and the two-level classification approach can be generalized to devices of other manufacturers (e.g., Apple Watch, Garmin) with adaptation.

\section{METHODS}

\subsection{Problem Formulation}

The objective of this study is to build a computational model for sleep staging based on processed data derived from Fitbit devices. We apply machine learning algorithms because they can discover hidden patterns in complex heterogeneous and high dimensional data (31). The nature of the problem is formulated as follows. Given $\boldsymbol{x}$ the feature space containing $M$ features, the $\boldsymbol{x}$ values are vectors in the form of $x^{v_{1}}, x^{v_{2}}, \ldots, x^{v_{M}}$, where $x^{v_{m}}$ is either nominal, discrete or continuous feature constructed from the input data. The $y$ values are class labels drawn from a discrete set of classes $\{1,2,3,4\}$, corresponding to deep sleep, light sleep, REM sleep and wakefulness. A two-level classification model constructs a cascading mapping function f: $y=f_{L_{-} I I}\left(f_{L_{-} I}(\mathbf{x})\right)$ based on $N^{T R}$ labeled training instances $\left(\mathbf{x}_{1}, y_{1}\right),\left(\mathbf{x}_{2}, y_{2}\right), \ldots,\left(\mathbf{x}_{N}^{T R}, y_{N}^{T R}\right)$, where $y$ belongs to $\{1,2,3,4\}$, so that the performance for $N^{T S}$ new unseen instances can be optimized. The level- 1 and level- 2 classifiers are denoted as $f_{L_{-} I}$ and $f_{L_{-} I I}$. Information related to the training sets and test sets are denoted using superscript notions $T R$ and $T S$, respectively.

In this study, the model performance was evaluated using multiple measures, which will be described in detail in section 2.5. As illustrated in Figure 1, the two-level model performs classification epoch-by-epoch, which is compliant with the standard epoch-wise sleep staging approach in clinical settings. For each epoch, the input data to the two-level model include the corresponding Fitbit labeled sleep stage, aggregated Fitbit sleep metrics for that day, heart rate data, and a few demographic features that can be measured using established psychometric instruments, such as the PSQI questionnaire (32). The output of the model for each epoch is one of the four sleep stages. Epochwise predicted sleep labels are aggregated to calculate the total duration of each sleep stage.

\subsection{Data Collection}

We conducted a three-night data collection experiment with 23 healthy adults (nine women; age $=24.8 \pm 4.4$ years) using a Fitbit Charge 2 and a medical sleep monitor named Sleep Scope. None of the participants had diagnosed sleep problems at the time of the study. Ethics approval was obtained from The University of Tokyo. Data collection was performed in participants' homes to ensure that the distribution of data and noise is representative of real situations in free-living environments. During the data 


\section{MODEL CONSTRUCTION}

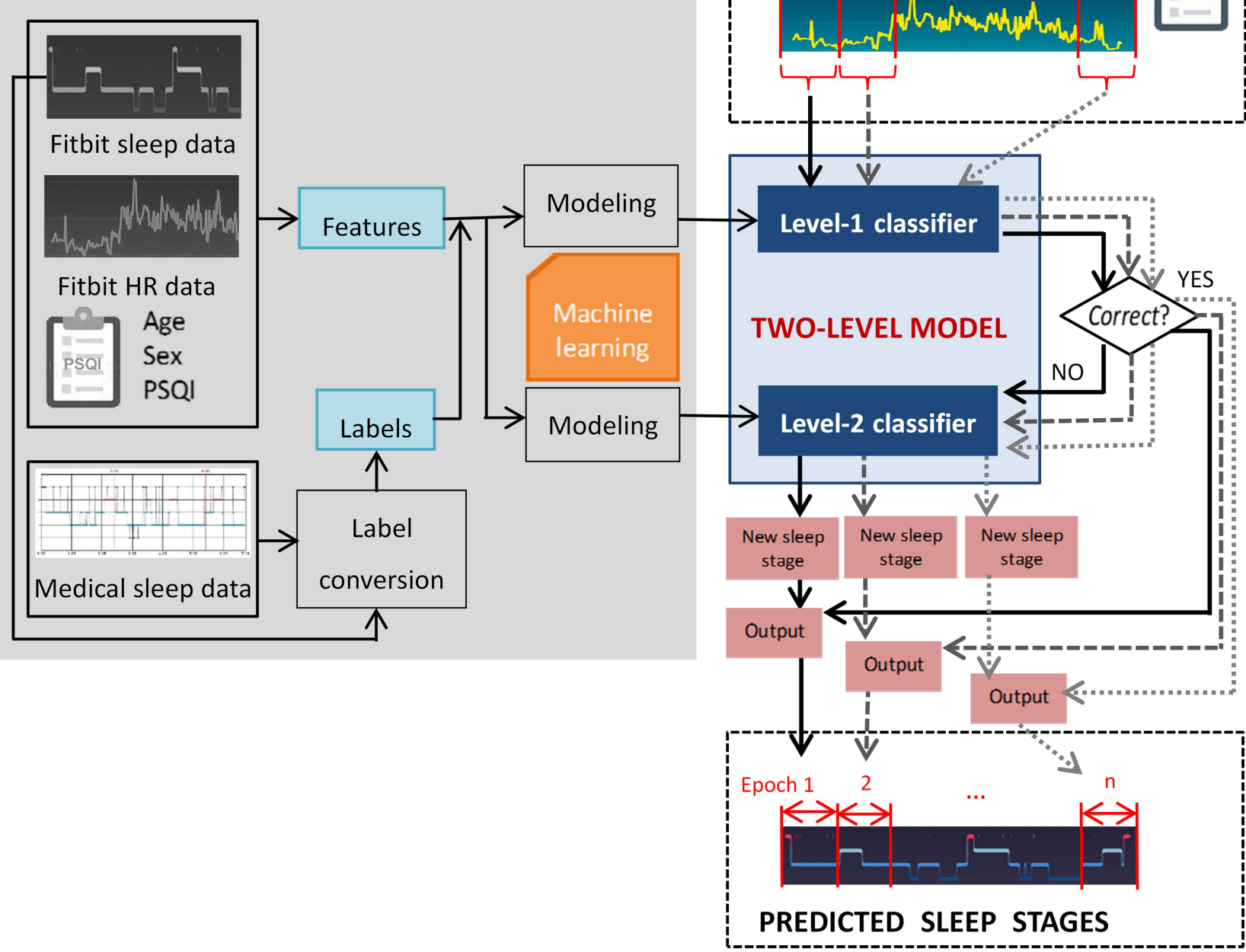

FIGURE 1 | Overview of the proposed two-level classification approach.

collection experiment, Fitbit Charge 2 was applied to the nondominant wrist. The electrodes of the Sleep Scope were placed in the middle of the forehead and behind one ear. Details of the two devices are available in Liang and Ploderer (12). Demographic information, such as age, sex, and subjective sleep quality measured by PSQI questionnaire (32) was also collected. The Fitbit data and demographic information are used to construct features while the medical data are used for labeling.

To mitigate the first night effect (33), we used the data of each participant's second night. Nonetheless, if any of the devices had more than $50 \%$ of data missing due to technical issues (e.g., electrodes peeling off, device running out of battery), the third night's data were used in place of the second night's. The first night's data were only used when both the second and the third night's data were discarded. Using a web app that we developed in our previous study (15), we retrieved Fitbit labeled sleep stages, aggregated daily sleep metrics, including total sleep time (TST), wake after sleep onset (WASO), sleep efficiency (SE) and the ratio of individual sleep stage, and heart rate data. The Fitbit labeled sleep stage data were retrieved at a granularity of 30 s. The Fitbit heart rate data were retrieved at a granularity of $1 \mathrm{~s}$ but in practice the granularity varied. The Sleep Scope generated analysis reports for each selected night, which consists of $30 \mathrm{~s}$-epoch-by-epoch sleep stage data. We wrote a C\# program to pre-process the Fitbit labeled sleep stage data, heart rate data and Sleep Scope labeled sleep stage data. All streams of data were firstly synchronized and missing data were handled using interpolation. The Fitbit labeled sleep stages, i.e., "deep sleep," "light sleep," "REM sleep," 
TABLE 1 | Features constructed for epoch $n$.

\begin{tabular}{|c|c|c|}
\hline Type & Feature & $\begin{array}{l}\text { Collection } \\
\text { method }\end{array}$ \\
\hline \multirow[t]{2}{*}{ Static } & Age, sex, Pittsburgh sleep quality index (PSQI) & $\begin{array}{l}\text { PSQI } \\
\text { Questionnaire }\end{array}$ \\
\hline & $\begin{array}{l}\text { Total sleep time (TST), wake after sleep onset } \\
\text { (WASO), sleep efficiency (SE), wake ratio, light } \\
\text { sleep ratio, deep sleep ratio, REM sleep ratio }\end{array}$ & Fitbit \\
\hline Dynamic & $\begin{array}{l}\text { ID of epoch } n \text {, Fitbit labeled sleep stage for } \\
\text { epoch }(n-3)-(n+3) \text {, average heart rate in } \\
\text { epoch } n \text {, heart rate change in epoch } n \text { vs. } \\
\text { epoch }(n-1) \text {, heart rate change in epoch } n+ \\
1 \text { vs. epoch } n\end{array}$ & Fitbit \\
\hline
\end{tabular}

and "wakefulness," were respectively mapped to the class " 1 ," " 2 ," "3," and "4." For Sleep Scope, "stage N3" was mapped to the class "1," "stage N1," and "stage N2" were mapped to the class "2," "stage R" was mapped to the class " 3 " and "stage W" was mapped to the class " 4 ."

\subsection{Feature Construction and Labeling}

We constructed 21 features as summarized in Table 1. All features can be derived from the data of Fitbit and PSQI questionnaire without relying on additional information. We selected these features because they have shown to associate to the accuracy of sleep staging $(28,34,35)$ and they are easy to derive from the available data. In contrast, other potential features that can be derived from the brainwave signals measured with the medical device and the raw acceleration data measured with Fitbit were not selected as these data are often not readily available in free-living environments.

We divide the features into static features and dynamic features. As shown in Table 1, static features refer to features that demonstrate no intra-individual variability for the selected night. These features include demographic characteristics (i.e., age, sex, and PSQI) and aggregated sleep metrics (i.e., TST, WASO, SE, wake ratio, light sleep ratio, deep sleep ratio, and REM sleep ratio). In contrast, dynamic features are derived from Fitbit time series data and they demonstrate variability from epoch to epoch. These features include epoch ID, Fitbit labeled sleep stage for the current epoch and three preceding and three succeeding epochs, average heart rate in the current epoch, heart rate change in the current epoch against the preceding epoch, and heart rate change in the succeeding epoch against the current epoch.

Figure 2 exemplifies the dataset preparation process. We first synchronized the time series data of Fitbit and the medical device to ensure that the data from the two devices matched epoch-wisely. Thereafter we constructed dynamic features from the time series data epoch-by-epoch and each epoch was fed to the dataset as an instance. Lastly, the static features and the labels for two levels were merged with the dynamic features. The same set of features were used at both levelI and level-II classifications. The ground truth labels were obtained epoch-wisely using the following conversion method. For level-I binary classification, the label for each instance was obtained through epoch-wise comparison between the Fitbit and the medical device. If the two devices agreed, the label was 0 (representing correct classification); otherwise, the label was 1 (representing misclassification). For level-II multi-class classification, the medical data was used as the labels. An example of label conversion is shown in Table 2.

\subsection{Model Construction}

We applied different machine learning algorithms at each level of classification. For level-I binary classification, we used three machine learning algorithms-Naïve Bayes (NB), random forest (RF), and support vector machine (SVM) with linear kernel. These algorithms were selected because of their simplicity, computational efficiency, and good performance on binary classification. For level-II classification which involves four classes, we applied the gradient boosting decision tree (XGBoost) because a preliminary analysis of model performance with default parameter values demonstrated their advantage over other widely used machine learning algorithms, such as artificial neural network. Model training of classifier-1 and classifier-2 were independently performed using the same dataset. For each level of classifier, model parameters were tuned through grid search with 10 -fold cross validation. Feature normalization was performed in the SVM algorithm.

A major challenge at the level-II classification lies in the imbalanced nature of the datasets, where the class " 2 " (indicating light sleep) significantly outnumbers other classes. The reason is that a night of normal human sleep typically consists of large portion of light sleep (40-60\%), while the portion of REM sleep (15-25\%), deep sleep (15-20\%), and wakefulness (5-15\%) are significantly smaller than light sleep (36). The hitch with the imbalanced nature of sleep data is that standard machine learning algorithms may bias toward the majority class and yield higher learning errors on the minority class. This is especially true for machine learning techniques that are sensitive to class distribution (37). To cope with this problem, we applied resampling techniques to balance different classes. We examined the effect of two simple resampling techniques: random up sampling and random down sampling. Random up sampling randomly generates data points to the minority classes so that the frequency of the minority classes is close to that of the majority one. Random down sampling randomly subsets the majority class to match the frequency of the minority classes. A preliminary study demonstrated the effectiveness of these techniques in enhancing model performance (38). It is worth mentioning that resampling was performed within cross-validation to ensure that only the training sets were resampled, and the test sets were not.

\subsection{Model Evaluation}

We obtained nine models with different combinations of resampling techniques and machine learning algorithms. Models are denoted as $L 1 \_L 2_{\text {resamp. }}$. For example, $N B \_X G B$ denotes the model that uses Naïve Bayes at level-I and XGBoost without resampling at level-II, and $N B \_X G B d$ denotes the model that uses Naïve Bayes at level-I and XGBoost with down sampling at level-II. We compare the performance of the nine two-level models with four baseline models, including the proprietary 


\section{Feature Construction}

\section{Label Conversion}

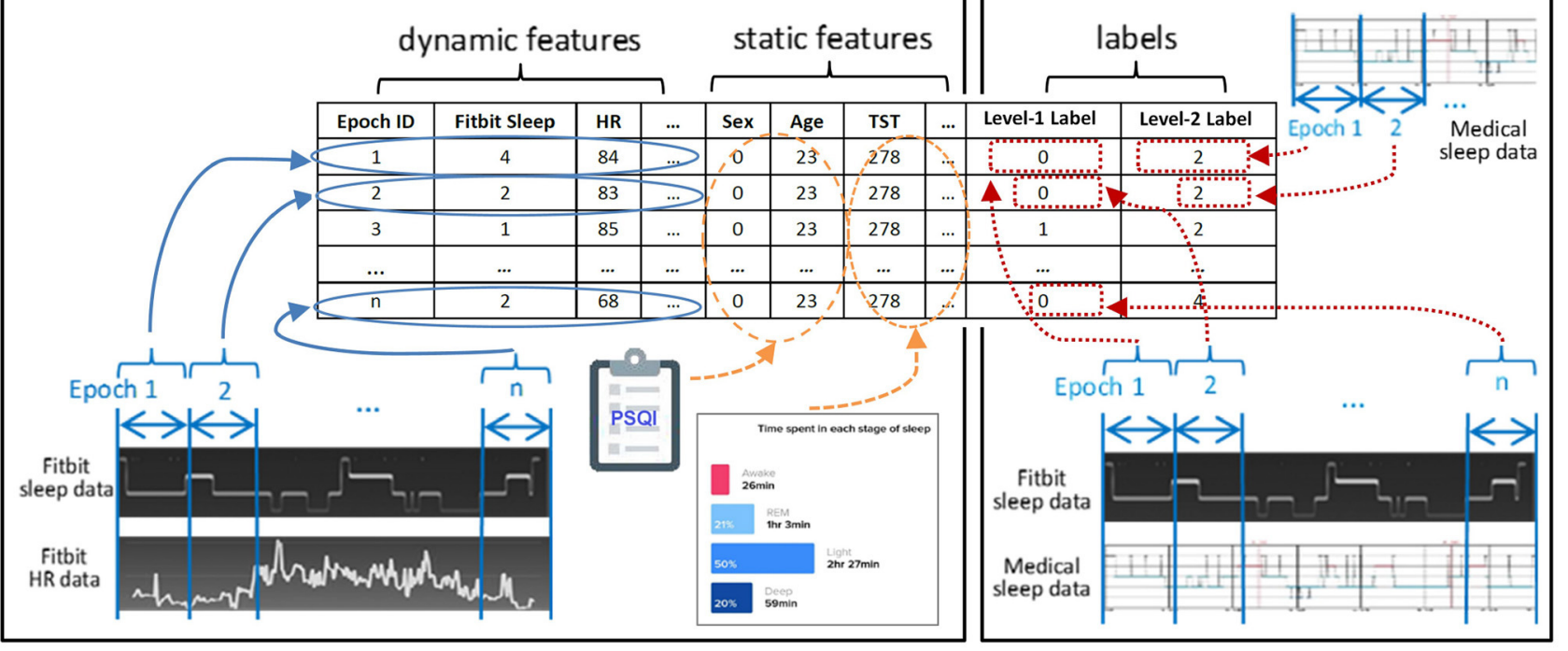

FIGURE 2 | Feature construction and label conversion.

TABLE 2 | An example of label conversion.

Epoch ID Fitbit Sleep Data Medical Sleep Data Level-I label Level-II label

\begin{tabular}{lllll}
\hline 1 & $4^{a}$ & 4 & 0 & 4 \\
2 & 4 & 4 & 0 & 4 \\
3 & 2 & 4 & 1 & 4 \\
4 & 2 & 4 & 1 & 4 \\
$\ldots$ & $\ldots$ & $\ldots$ & $\ldots$ & $\ldots$ \\
$\mathrm{N}-1$ & 3 & 2 & 1 & 2 \\
$\mathrm{~N}$ & 3 & 3 & 0 & 3 \\
\hline
\end{tabular}

$a_{1}$ = deep sleep, 2 = light sleep, 3 = REM sleep, 4 = wakefulness.

Fitbit model (denoted as Fitbit) and three one-level models (denoted as $X G B, X G B u$, and $X G B d$ ). The three one-level models attempt to reclassify all Fitbit labeled sleep stage epochs through four-class classification instead of selective correction as the twolevel models do. These three baseline models all use XGBoost algorithm but with different resampling strategies.

We evaluate model performance using nested leave-onesubject-out cross validation (LOSO-CV) (39). There are two practical reasons underlying the necessity of using this strategy. First, due to the chronological nature of sleep data, a model should be tested on a whole night of sleep data from one person, not over a mix of epochs from different people. Second, since class distribution demonstrated interpersonal variations, it is important to check the performance of the models on test set with possible data shift, which refers to the cases where training and test data follow different distribution and is especially relevant to imbalanced classification $(40,41)$. As illustrated in Figure 3, the nested LOSO-CV operates in an iterated manner. In each iteration, the data of one participant are held out as the test set, while the data of the remaining 22 participants are merged and used as the training set. A model is tuned on the training set through intensive grid search using 10 -fold cross validation. Once the model is fixed on the training set, it was evaluated on the test set. The nested-evaluation process iterates 23 times with each subject's data being kept out as test set once. We then averaged the performance over all the 23 iterations.

We adopt two types of performance measures-microscopic performance metrics and macroscopic performance metrics-to thoroughly evaluate the two-level models. These measures cover not only epoch-wise comparison between the models and the ground truth (with microscopic measures) but also comparison on aggregated sleep metrics (with macroscopic measures).

\subsubsection{Microscopic Measures}

Microscopic measures are calculated based on epoch-wise comparison between model predicted value and the true value of each epoch. These measures include overall per-epoch accuracy, Cohen's Kappa, multi-class Mathew's correlation coefficient (MMCC), confusion matrix, correcting power (CP) and overcorrecting rate (OR). The per-epoch accuracy and Kappa have been widely used to evaluate the performance of automatic sleep staging algorithms in sleep research. Despite that they may not be the most appropriate performance measure for imbalanced classification, we use them as complementary metric to facilitate cross-study comparison. On the other hand, MMCC is widely accepted as a more appropriate performance measure for imbalanced multi-class classification as it considers the accuracy and error rates on all classes and involves all elements of a confusion matrix $(42,43)$. The $\mathrm{CP}$ and $\mathrm{OR}$ are original measures specific to our approach for probing deeper into the behavior of the two-level models. While an ideal model would 


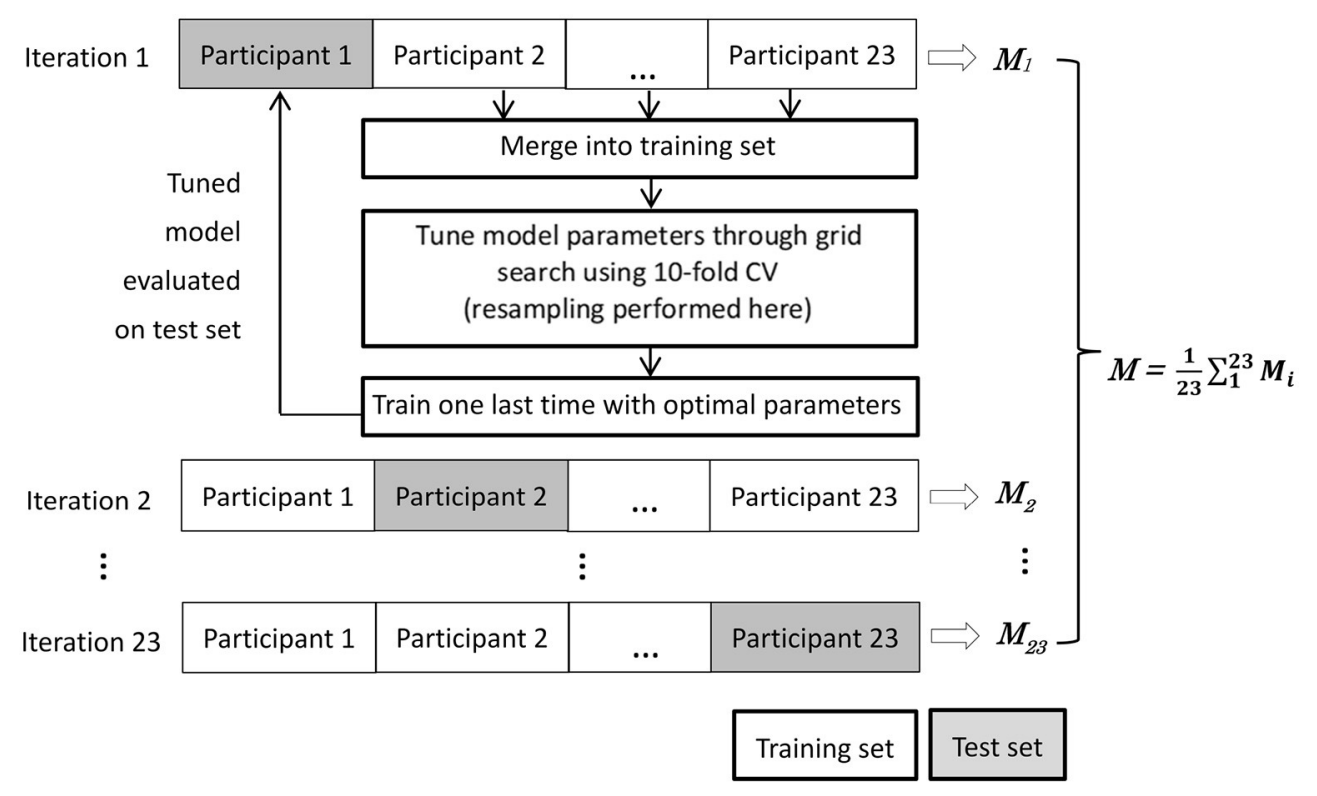

FIGURE 3 | Nested leave-one-subject-out cross validation (LOSO-CV). The $\boldsymbol{M}$ denotes the set of performance measures.

be characterized by high $\mathrm{CP}$ and low $\mathrm{OR}$, in practice it is only feasible to achieve a good trade-off between the two metrics.

- Overall per-epoch accuracy: The number of correctly predicted sleep epochs vs. the total number of epochs. Despite that this metric is not considered as a legitimate performance measure for imbalanced classification in the machine learning community, we use it as a complementary metric that facilitates cross-study comparison.

- Cohen's Kappa: An indicator of model quality by taking into account how much agreement between predicted and true values would be attributed to chance. According to (44), a value $<0$ indicates no agreement, $0-0.40$ as poor, $0.40-0.75$ as fair to good, $>0.75$ as excellent. Higher value indicates better performance.

- Multi-class Matthew's correlation coefficient (MMCC): A single metric that summarizes a confusion matrix and has been considered as one of the best performance measure for multiclass imbalanced classification. A value of -1 and 1 represent perfect misclassification and perfect classification, and a value of 0 indicates random guess $(45,46)$.

- Confusion matrix: A table that summarizes the prediction accuracy and error rate on individual sleep stages. Each row represents an actual sleep stage, while each column represents a predicted sleep stage.

- Correcting power (CP): The ratio of epochs that are mislabeled by Fitbit and are corrected by the two-level model. Higher value of $\mathrm{CP}$ means that more Fitbit misclassified epochs are corrected by the model and hence better model performance.

- Over-correcting rate (OR): The ratio of epochs that are correctly labeled by Fitbit but are misclassified by the two-level model. Lower value of $\mathrm{OR}$ indicates better model performance.

$$
\Delta=\frac{\mathbf{M}_{\text {proposal }}-\mathbf{M}_{\text {fitbit }}}{\mathbf{M}_{\text {fitbit }}} \times 100 \%
$$

The performance improvement of the two-level models over Fitbit, which is denoted as $\Delta$, is computed using Equation (1), where $\mathbf{M}_{\text {proposal }}$ and $\mathbf{M}_{\text {fitbit }}$ denote the performance (e.g., accuracy, Kappa, and MMCC) of the two-level models and Fitbit, respectively. Two-tailed $t$-test is applied to examine if statistically significance difference exist between the average performance of the two-level models and that of the Fitbit.

We also perform Pearson's correlation analysis between the performance measures and the class imbalance or the dataset shift to investigate the effect of dataset characteristics on model performance. The Shannon's diversity index (47) is used to quantify the imbalance of individual sleep stage in the test set of each iteration in the LOSO-CV process. A lower value of the Shannon's diversity index implies more imbalance of classes. The Anderson-Darling (AD) statistic (48) is adopted to quantify the difference in sleep stage distribution between a training set and the corresponding test set in each iteration of the LOSO-CV process. A higher value of the AD statistic indicates larger shift between a training set and the corresponding test set.

\subsubsection{Macroscopic Measures}

Macroscopic measures are calculated by comparing the predicted values and true values on aggregated sleep metrics including the total duration of light sleep, deep sleep, REM sleep, and wakefulness. The following metrics are adopted.

- Bland-Altman (BA) plot (49): A plot that illustrates the difference between two methods as a function of the mean of these methods. The visual examination of the BA plot allows for an evaluation of the global agreement between 
TABLE 3 | Demographic information and sleep characteristics.

\begin{tabular}{|c|c|c|c|c|c|c|}
\hline & Age & PSQI & $\begin{array}{l}\text { Wakefulness } \\
\text { (min) }\end{array}$ & $\begin{array}{l}\text { Light sleep } \\
\qquad(\min )\end{array}$ & $\begin{array}{l}\text { Deep sleep } \\
\text { (min) }\end{array}$ & $\begin{array}{l}\text { REM sleep } \\
\text { (min) }\end{array}$ \\
\hline All $(N=23)$ & $24.3 \pm 2.7$ & $4.3 \pm 2.3$ & $55.7 \pm 52.9$ & $499.1 \pm 149.0$ & $45.1 \pm 64.5$ & $177.4 \pm 66.1$ \\
\hline Male $(N=14)$ & $24.4 \pm 2.8$ & $4.6 \pm 2.7$ & $59.1 \pm 40.4$ & $537.1 \pm 153.1$ & $32.4 \pm 50.7$ & $171.8 \pm 68.6$ \\
\hline
\end{tabular}

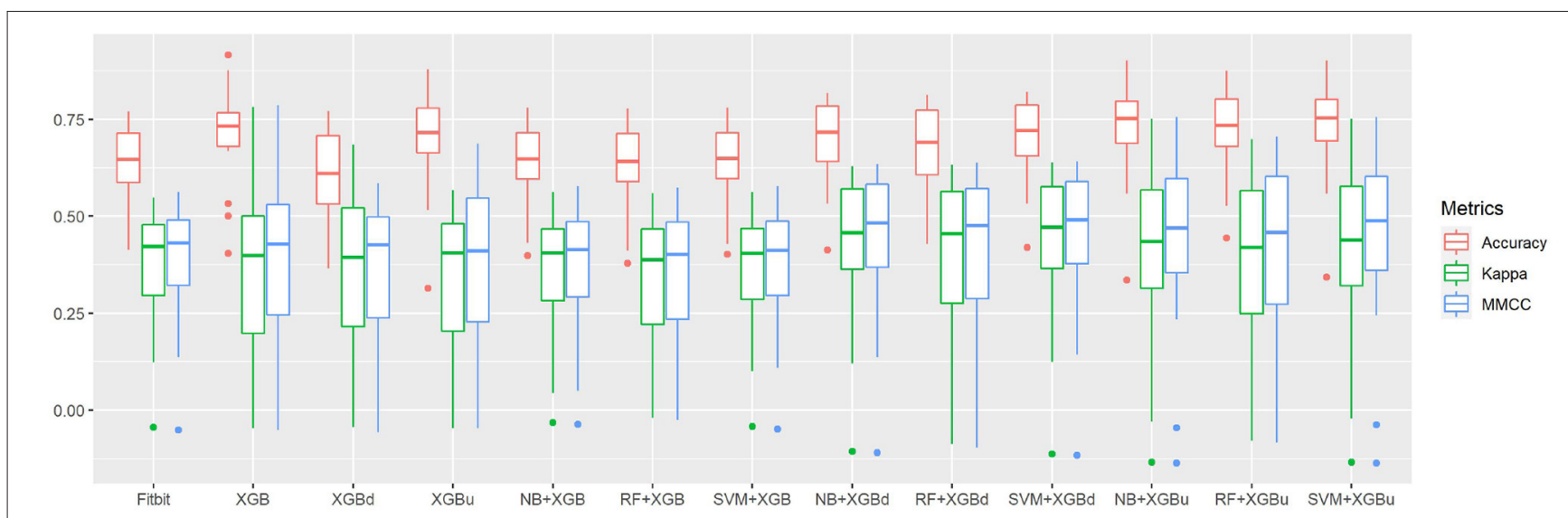

FIGURE 4 | The overall per-epoch accuracy, Kappa and multi-class Matthew's correlation coefficient (MMCC) of sleep staging models.

the model and the ground truth. The biases and lower and upper level of agreement (LOA) are calculated. Two-tailed one sample $t$-test is performed to examine whether the biases are statistically different from 0 . A positive bias indicates that the model underestimated a sleep metric against the ground truth, while a negative bias indicates overestimation. The LOAs are computed as bias \pm 1.96 standard deviation of the differences. Linear fitting with statistical test is performed to examine whether there is a significant tend in errors as a function of the magnitude of the measured value.

- Mean normalized absolute bias (MAB): An average over the normalized absolute values of the bias (i.e., the average discrepancy between a model and the ground truth) of each sleep stage. The MAB of model $j$ is calculated using Equation (2), where $\left|e_{i, j}\right|$ denotes the absolute value of the bias of model $j$ for sleep stage $i, \min \left|e_{i}\right|$ and $\max \left|e_{i}\right|$ denote the minimum and maximum absolute bias of all models for sleep stage $i$, and $W, L, D, R$ denote wakefulness, light sleep, deep sleep, and REM sleep, respectively.

$$
M A B_{j}=\frac{1}{4} \sum_{i \in\{W, L, D, R\}} \frac{\left|e_{i, j}\right|-\min \left|e_{i}\right|}{\max \left|e_{i}\right|-\min \left|e_{i}\right|}
$$

\section{RESULTS}

\subsection{Descriptive Statistics}

The processed dataset consists of 23 nights of sleep data-one night from each participant. Among all the nights of data, five cases used the first night, 13 cases the second night, and five cases the third night. The total sleep time as measured by the medical device ranges from 4.0 to $9.8 \mathrm{~h}$. Complying with the practice in sleep research, each night of sleep was divided into 30 s-epochs, yielding 480-1,176 epochs for individual participant. The demographic information and the sleep characteristics as measured with the medical device and the PSQI are summarized in Table 3. It is worth noting that 12 participants (five females) had a PSQI score higher than or equal to 5, indicating perceived poor sleep quality at the time of the study. These participants referred to their academic stress or the sultry weather as the main reasons of their temporarily poor sleep. In what follows we describe the micro-level and macro-level performance of the models.

\subsection{Microscopic Performance}

Figure 4 shows the box plots of the overall per-epoch accuracy, Cohen's Kappa and multi-class Matthew's correlation coefficient (MMCC). The two-level approach demonstrates the best performance when SVM and XGBoost with up sampling are used, respectively for the level-I and level-II classification, yielding an overall per-epoch accuracy of $0.731 \pm 0.119$ (a $3.4-21.2 \%$ increase compared to the four baseline models), Cohen's kappa of $0.433 \pm 0.212$ (a 14.4-24.1\% increase) and MMCC of $0.451 \pm 0.214$ (a 10.9-16.6\% increase). Table 4 presents the performance comparison between Fitbit and the best top six models together with the hypothesis testing results. Two-tailed $t$-test shows that most two-level models achieved statistically significant improvement of per-epoch accuracy. The improvement in Kappa and MMCC was not significant, which is likely due to the relatively small sample size $(N=23)$. A 
TABLE 4 | Comparison between Fitbit and the best two-level models on microscopic performance measures.

\begin{tabular}{lccc}
\hline & Per-epoch accuracy & Kappa & MMCC \\
\hline Fitbit & $0.638 \pm 0.100$ & $0.371 \pm 0.148$ & $0.387 \pm 0.151$ \\
$\mathrm{NBd}+\mathrm{XGBd}$ & $\mathbf{0 . 7 0 1} \pm \mathbf{0 . 0 9 8 ^ { \star , a }}$ & $0.422 \pm 0.175$ & $0.437 \pm 0.177$ \\
$\mathrm{RF}+\mathrm{XGBd}$ & $0.681 \pm 0.109$ & $0.405 \pm 0.187$ & $0.419 \pm 0.191$ \\
$\mathrm{SVM}+\mathrm{XGBd}$ & $\mathbf{0 . 7 0 4} \pm \mathbf{0 . 0 9 7}^{*}$ & $0.427 \pm 0.178$ & $0.439 \pm 0.180$ \\
$\mathrm{NBd}+\mathrm{XGBu}$ & $\mathbf{0 . 7 2 8} \pm \mathbf{0 . 1 2 3}^{* *}$ & $0.427 \pm 0.214$ & $0.445 \pm 0.215$ \\
$\mathrm{RF}+\mathrm{XGBu}$ & $\mathbf{0 . 7 2 2} \pm \mathbf{0 . 1 0 9}^{* *}$ & $0.414 \pm 0.206$ & $0.437 \pm 0.200$ \\
$\mathrm{SVM}+\mathrm{XGBu}$ & $\mathbf{0 . 7 3 1} \pm \mathbf{0 . 1 1 9}^{* *}$ & $0.433 \pm 0.212$ & $0.451 \pm 0.214$
\end{tabular}

a Bold indicates statistically significant difference compared to Fitbit. ${ }^{*} p<0.05$; ${ }^{* *} p<0.01$.

comparison among the three baseline models (i.e., XGB, XGBd, and $\mathrm{XGBu}$ ) shows that resampling alone does not improve model performance. Adding one level of binary classification to the baseline model with no resampling (i.e., $\mathrm{NB}+\mathrm{XGB}, \mathrm{RF}$ $+\mathrm{XGB}$, and $\mathrm{SVM}+\mathrm{XGB}$ ) reduces the variability but not the average of model performance. In contrast, adding one level of binary classification to the baseline model with resampling successfully improved the model performance. This indicates that resampling at level-II is essential to the performance of the two-level approach.

The confusion matrix for individual sleep stage is provided in Table 5. RF + XGBd achieved the best sleep stage wise performance and improved the average accuracy for both light sleep (by $12.1 \%$ compared to Fitbit) and REM sleep (by $14.0 \%$ ), while minimize the sacrifice on the classification accuracy for deep sleep. In general, down sampling at the level-II classification helped achieved a more balanced performance, whereas up sampling had trivial effect on the overall tendency of the final outcome. With either XGB or XGBu being used for the levelII classification, the models tend to have the best average accuracy for light sleep but deteriorated accuracy for deep sleep and wakefulness. The most common misclassifications were deep/light (two-level model: 42.9-85.3\%; Fitbit: 29.1\%), REM/light (two-level model: 22.9-41.9\%; Fitbit: $31.7 \%$ ), and wake/light mislabeling (two-level model: 25.7-71.3\%; Fitbit: $50.3 \%)$. Figure 5 illustrates the correcting power (CP) and overcorrecting rate (OR) of the models. The best trade-off between $\mathrm{CP}$ and $\mathrm{OR}$ is achieved when up sampling was applied at the level-II classification.

Table 6 provides the results of the Pearson's correlation analysis between the microscopic performance measures and the Shannon's diversity index of the test sets. Per-epoch accuracy, Kappa and MMCC are in general positively correlated to the Shannon's diversity index. Such correlation is moderate but statistically significant for Fitbit, NB + XGB, RF + XGB, SVM + $\mathrm{XGB}, \mathrm{RF}+\mathrm{XGBd}$, which implies improved model performance when class distribution in the test sets are more balanced. CP and OR may be weakly or moderately correlated to Shannon's diversity index, but with no statistical significance. Table 7 shows that the AD statistic is negatively correlated to the perepoch accuracy of almost all models. The correlation strength ranges from moderate to strong and with statistical significance, indicating that prediction errors are more pronounced when
TABLE 5 | Confusion matrix for sleep stages (\%).

\begin{tabular}{|c|c|c|c|c|c|}
\hline & & \multicolumn{4}{|c|}{ Ground truth } \\
\hline & & Light sleep & Deep sleep & REM sleep & Wakefulness \\
\hline \multirow{13}{*}{$\begin{array}{l}\text { Light } \\
\text { sleep }\end{array}$} & Fitbit & 69.3 & 29.1 & 31.7 & 50.3 \\
\hline & XGB & 87.0 & 83.0 & 41.9 & 64.4 \\
\hline & XGBd & 61.7 & 42.9 & 22.9 & 25.7 \\
\hline & XGBu & 85.1 & 78.7 & 41.1 & 62.0 \\
\hline & $\mathrm{NB}+\mathrm{XGB}$ & 90.9 & 85.3 & 37.3 & 70.2 \\
\hline & $R F+X G B$ & 90.2 & 82.6 & 40.1 & 65.5 \\
\hline & SVM + XGB & 91.2 & 85.4 & 37.1 & 71.3 \\
\hline & $\mathrm{NB}+\mathrm{XGBd}$ & 81.0 & 52.6 & 33.8 & 53.4 \\
\hline & $R F+X G B d$ & 77.7 & 49.9 & 33.1 & 46.1 \\
\hline & $S V M+X G B d$ & 81.5 & 55.5 & 33.9 & 54.8 \\
\hline & $\mathrm{NB}+\mathrm{XGBu}$ & 89.4 & 79.4 & 36.8 & 68.6 \\
\hline & $\mathrm{RF}+\mathrm{XGBu}$ & 88.7 & 77.9 & 39.8 & 63.4 \\
\hline & SVM + XGBu & 89.6 & 81.0 & 36.3 & 69.3 \\
\hline \multirow{13}{*}{$\begin{array}{l}\text { Deep } \\
\text { sleep }\end{array}$} & Fitbit & 21.6 & 60.9 & 3.5 & 6.5 \\
\hline & XGB & 1.1 & 4.4 & 0.0 & 0.4 \\
\hline & XGBd & 11.2 & 39.6 & 1.7 & 2.3 \\
\hline & XGBu & 1.8 & 9.3 & 0.0 & 0.4 \\
\hline & $\mathrm{NB}+\mathrm{XGB}$ & 1.0 & 3.8 & 0.6 & 0.6 \\
\hline & $\mathrm{RF}+\mathrm{XGB}$ & 1.2 & 5.4 & 0.2 & 0.5 \\
\hline & SVM + XGB & 0.8 & 3.7 & 0.0 & 0.5 \\
\hline & $\mathrm{NB}+\mathrm{XGBd}$ & 9.1 & 36.4 & 1.5 & 1.6 \\
\hline & $\mathrm{RF}+\mathrm{XGBd}$ & 9.5 & 36.3 & 0.9 & 1.7 \\
\hline & SVM + XGBd & 8.9 & 34.1 & 0.7 & 1.2 \\
\hline & $\mathrm{NB}+\mathrm{XGBu}$ & 1.8 & 9.4 & 0.7 & 0.6 \\
\hline & $\mathrm{RF}+\mathrm{XGBu}$ & 2.0 & 10.7 & 0.1 & 0.5 \\
\hline & SVM + XGBu & 1.6 & 8.7 & 0.0 & 0.4 \\
\hline \multirow{13}{*}{$\begin{array}{l}\text { REM } \\
\text { sleep }\end{array}$} & Fitbit & 5.9 & 2.9 & 59.6 & 8.1 \\
\hline & XGB & 9.5 & 5.7 & 54.5 & 12.0 \\
\hline & XGBd & 14.2 & 7.1 & 63.9 & 18.8 \\
\hline & XGBu & 8.6 & 4.9 & 54.6 & 12.9 \\
\hline & $\mathrm{NB}+\mathrm{XGB}$ & 6.9 & 4.0 & 59.8 & 9.3 \\
\hline & $R F+X G B$ & 6.5 & 5.0 & 56.8 & 11.3 \\
\hline & SVM + XGB & 6.9 & 4.0 & 60.7 & 9.3 \\
\hline & $\mathrm{NB}+\mathrm{XGBd}$ & 6.6 & 3.6 & 60.4 & 10.6 \\
\hline & $\mathrm{RF}+\mathrm{XGBd}$ & 7.7 & 5.5 & 59.2 & 12.3 \\
\hline & $S V M+X G B d$ & 6.7 & 3.3 & 61.0 & 10.6 \\
\hline & $\mathrm{NB}+\mathrm{XGBu}$ & 7.1 & 4.2 & 60.0 & 10.3 \\
\hline & $\mathrm{RF}+\mathrm{XGBu}$ & 6.4 & 4.5 & 56.3 & 11.0 \\
\hline & $\mathrm{SVM}+\mathrm{XGBu}$ & 7.1 & 3.6 & 60.9 & 10.2 \\
\hline \multirow[t]{13}{*}{ Wakefulness } & Fitbit & 3.2 & 2.7 & 5.3 & 35.0 \\
\hline & XGB & 2.4 & 2.6 & 3.6 & 23.1 \\
\hline & XGBd & 12.9 & 6.1 & 11.5 & 53.2 \\
\hline & XGBu & 4.5 & 2.7 & 4.4 & 24.7 \\
\hline & $\mathrm{NB}+\mathrm{XGB}$ & 1.2 & 2.4 & 2.3 & 20.0 \\
\hline & $R F+X G B$ & 2.0 & 2.4 & 2.9 & 22.7 \\
\hline & SVM + XGB & 1.2 & 2.4 & 2.2 & 19.0 \\
\hline & $\mathrm{NB}+\mathrm{XGBd}$ & 3.3 & 3.1 & 4.3 & 34.4 \\
\hline & $\mathrm{RF}+\mathrm{XGBd}$ & 5.1 & 4.0 & 6.8 & 39.9 \\
\hline & $S V M+X G B d$ & 2.9 & 2.8 & 4.4 & 33.4 \\
\hline & $\mathrm{NB}+\mathrm{XGBu}$ & 1.7 & 2.4 & 2.5 & 20.5 \\
\hline & $\mathrm{RF}+\mathrm{XGBu}$ & 2.9 & 2.5 & 3.7 & 25.0 \\
\hline & $\mathrm{SVM}+\mathrm{XGBu}$ & 1.6 & 2.4 & 2.8 & 20.0 \\
\hline
\end{tabular}

The bold values indicate the percentage of correctly classified epochs for each sleep stage by different models. 


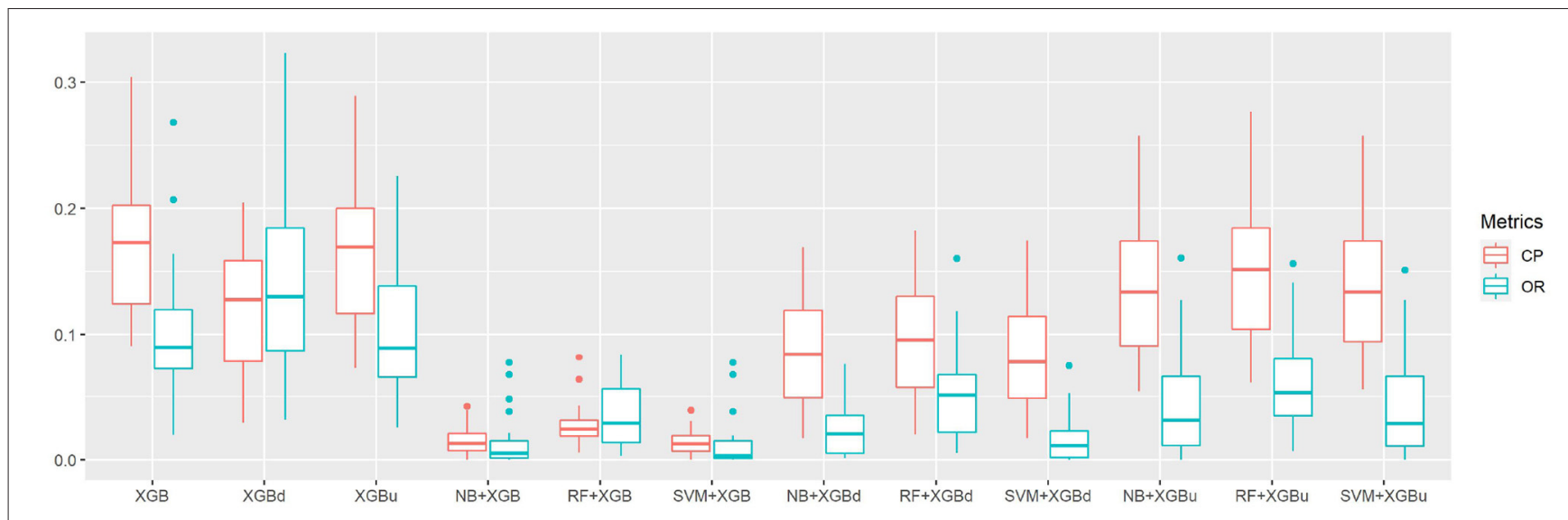

FIGURE 5 | The correction power (CP) and over-correction rate (OR) of sleep staging models.

TABLE 6 | Pearson's correlation coefficients between model performance and Shannon's diversity index (SDI) of test sets.

\begin{tabular}{|c|c|c|c|c|c|}
\hline Model & $r_{\text {accuracy }}^{\text {SDI }}$ & $r_{\text {kappa }}^{\text {SDI }}$ & $r_{m m c c}^{S D I}$ & $r_{c p}^{S D I}$ & $r_{o r}^{S D I}$ \\
\hline Fitbit & $0.46^{* a}$ & $0.48^{*}$ & $0.51^{*}$ & / & / \\
\hline XGB & 0.35 & 0.36 & 0.32 & -0.06 & -0.06 \\
\hline XGBd & 0.36 & $0.45^{*}$ & 0.40 & -0.31 & -0.23 \\
\hline XGBu & 0.38 & 0.37 & $0.41^{*}$ & -0.14 & -0.25 \\
\hline$N B+X G B$ & $0.46^{*}$ & $0.47^{*}$ & $0.48^{*}$ & 0.04 & -0.08 \\
\hline$R F+X G B$ & $0.46^{*}$ & $0.51^{*}$ & $0.51^{*}$ & -0.23 & -0.30 \\
\hline SVM + XGB & $0.45^{*}$ & $0.45^{*}$ & $0.46^{*}$ & 0.12 & -0.03 \\
\hline $\mathrm{NB}+\mathrm{XGBd}$ & 0.35 & -0.06 & -0.04 & 0.02 & -0.00 \\
\hline$R F+X G B d$ & $0.43^{*}$ & $0.46^{*}$ & $0.46^{*}$ & -0.12 & -0.24 \\
\hline SVM + XGBd & 0.33 & 0.36 & 0.37 & -0.08 & 0.33 \\
\hline $\mathrm{NB}+\mathrm{XGBu}$ & 0.39 & -0.13 & -0.12 & -0.04 & 0.25 \\
\hline $\mathrm{RF}+\mathrm{XGBu}$ & 0.38 & $0.46^{*}$ & $0.43^{*}$ & -0.14 & -0.16 \\
\hline SVM + XGBu & 0.37 & 0.40 & 0.40 & 0.00 & -0.04 \\
\hline
\end{tabular}

${ }^{a}$ Bold indicates statistically significant correlation. ${ }^{*} p<0.05$.

there is larger data shift between training set and test set. Kappa and MMCC are not as strongly correlated to the AD statistic as per-epoch accuracy. CP may be moderately and negatively correlated to the $\mathrm{AD}$ statistic but the relations are not significant. OR shows strong and positive correlation to the $\mathrm{AD}$ statistic for $\mathrm{XGBu}$ and $\mathrm{SVM}+\mathrm{XGBu}$, indicating increased over-correction rate for these models when there is large drift in data distribution between training set and test set.

\subsection{Macroscopic Performance}

The Bland-Altman plots constructed for the individual sleep stage are provided in Figures 6-9. Figure 6 shows that the twolevel model RF + XGBd (mean bias: 4.7 min, bias to limit: 194.9 $\min ; t=0.224, p=0.825$ ) agreed well to the ground truth for light sleep. Two other models, NBd + XGBd (mean bias: $-19.7 \mathrm{~min}$, bias to limit: $353.5 \mathrm{~min}$; $t=-0.525, p=0.605)$ and SVM $+\mathrm{XGBd}$
TABLE 7 | Pearson's correlation coefficients between model performance and Anderson-Darling (AD) statistic.

\begin{tabular}{|c|c|c|c|c|c|}
\hline Model & $r_{\text {accuracy }}^{A D}$ & $r_{\text {kappa }}^{A D}$ & $r_{m m c c}^{A D}$ & $r_{c p}^{A D}$ & $r_{o r}^{A D}$ \\
\hline XGB & $-0.65^{* * * a}$ & -0.36 & -0.36 & -0.31 & 0.40 \\
\hline XGBd & -0.40 & $-0.45^{*}$ & -0.28 & -0.07 & 0.10 \\
\hline XGBu & $-0.72^{* * *}$ & -0.26 & $-0.44^{*}$ & -0.31 & $0.64^{* * *}$ \\
\hline $\mathrm{NB}+\mathrm{XGB}$ & $-0.43^{* *}$ & -0.21 & -0.21 & -0.05 & 0.11 \\
\hline$R F+X G B$ & $-0.42^{*}$ & -0.23 & -0.22 & -0.08 & 0.05 \\
\hline$S V M+X G B$ & $-0.44^{*}$ & -0.21 & -0.21 & -0.00 & 0.12 \\
\hline $\mathrm{NB}+\mathrm{XGBd}$ & $-0.65^{* * *}$ & 0.10 & 0.11 & 0.17 & 0.04 \\
\hline$R F+X G B d$ & $-0.54^{* *}$ & -0.29 & -0.28 & -0.37 & 0.04 \\
\hline$S V M+X G B d$ & $-0.64^{* *}$ & -0.31 & -0.30 & -0.39 & 0.22 \\
\hline $\mathrm{NB}+\mathrm{XGBu}$ & $-0.75^{* * *}$ & 0.05 & 0.06 & 0.03 & 0.04 \\
\hline $\mathrm{RF}+\mathrm{XGBu}$ & $-0.67^{* * *}$ & -0.37 & -0.34 & -0.36 & 0.36 \\
\hline $\mathrm{SVM}+\mathrm{XGBu}$ & $-0.76 * * *$ & $-0.47^{*}$ & $-0.46^{*}$ & -0.36 & $0.81^{* * *}$ \\
\hline
\end{tabular}

${ }^{a}$ Bold indicates statistically significant correlation. ${ }^{*} p<0.05 ;{ }^{* *} p<0.01 ;{ }^{* * *} p<0.001$.

(mean bias: $-20.9 \mathrm{~min}$, bias to limit: $190.8 \mathrm{~min} ; t=-1.030, p$ $=0.314$ ), also had smaller mean bias compared to Fitbit (mean bias: $55.9 \mathrm{~min}$, bias to limit: $192.0 \mathrm{~min} ; t=2.737, p=0.012$ ). Statistical test on the linear fitting between model difference and model mean showed no significant trend in all models.

All two-level models have better agreement to the ground truth for deep sleep compared to the baseline models. The best agreement was achieved by SVM + XGBd (mean bias: $-15.3 \mathrm{~min}$, bias to limit: $154 \mathrm{~min} ; t=-0.929, p=0.363$ ), followed by RF + XGBd (mean bias: $-18.5 \mathrm{~min}$, bias to limit: $153.8 ; t=-1.129, p$ $=0.271$ ) and NBd + XGBd (mean bias: $-18.8 \mathrm{~min}$; bias to limit: $178.0 \mathrm{~min} ; t=-0.991, p=0.332)$. These three models together with Fitbit demonstrated no statistically significant trend. In contrast, a positive trend was observed in other models. Better agreement was observed for nights with shorter deep sleep, and on nights when there was longer deep sleep the models were more variable. We also noticed that the scatter around the bias line gets larger as the average gets higher. 


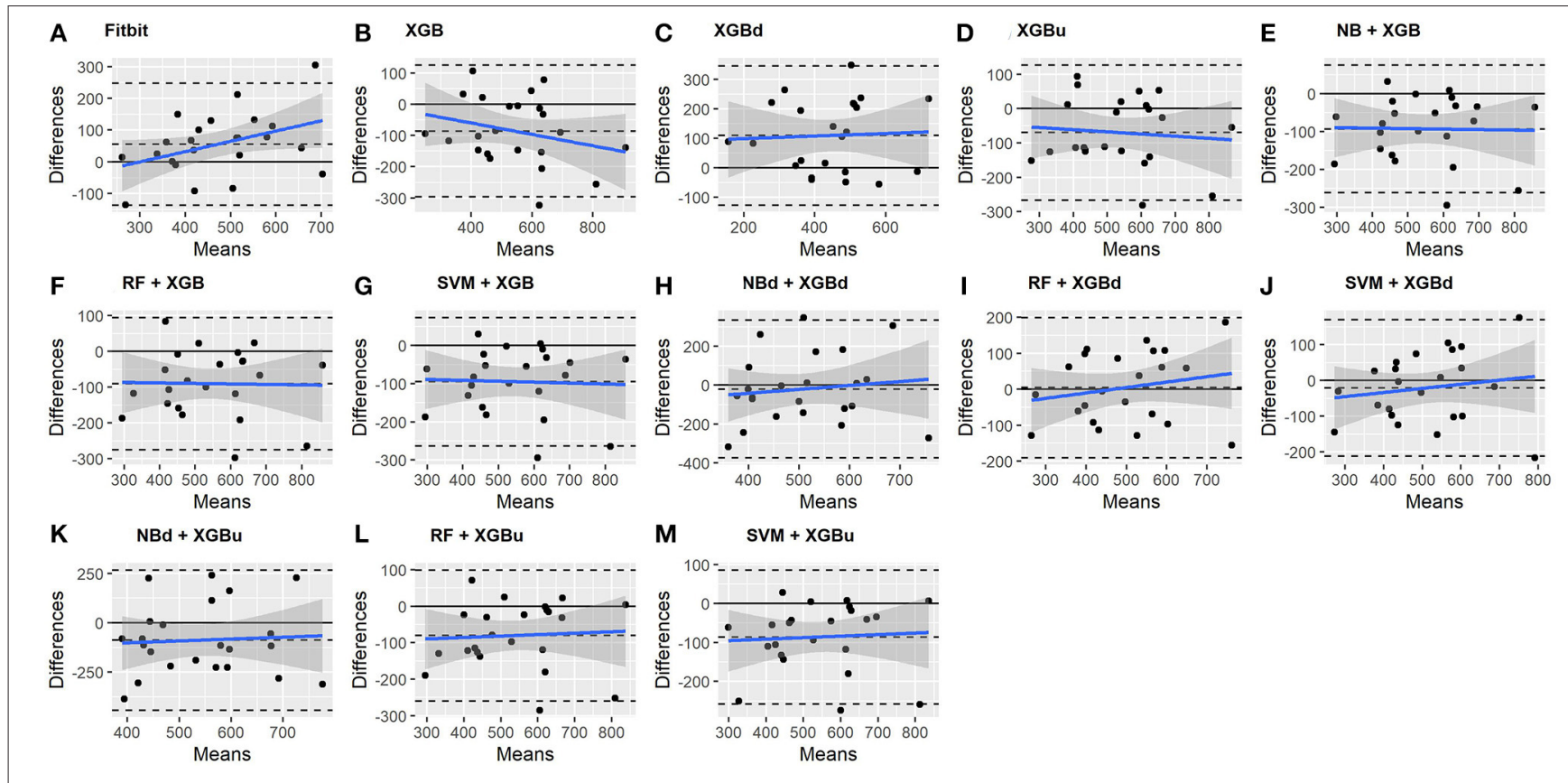

FIGURE 6 | Bland-Altman plots for light sleep.

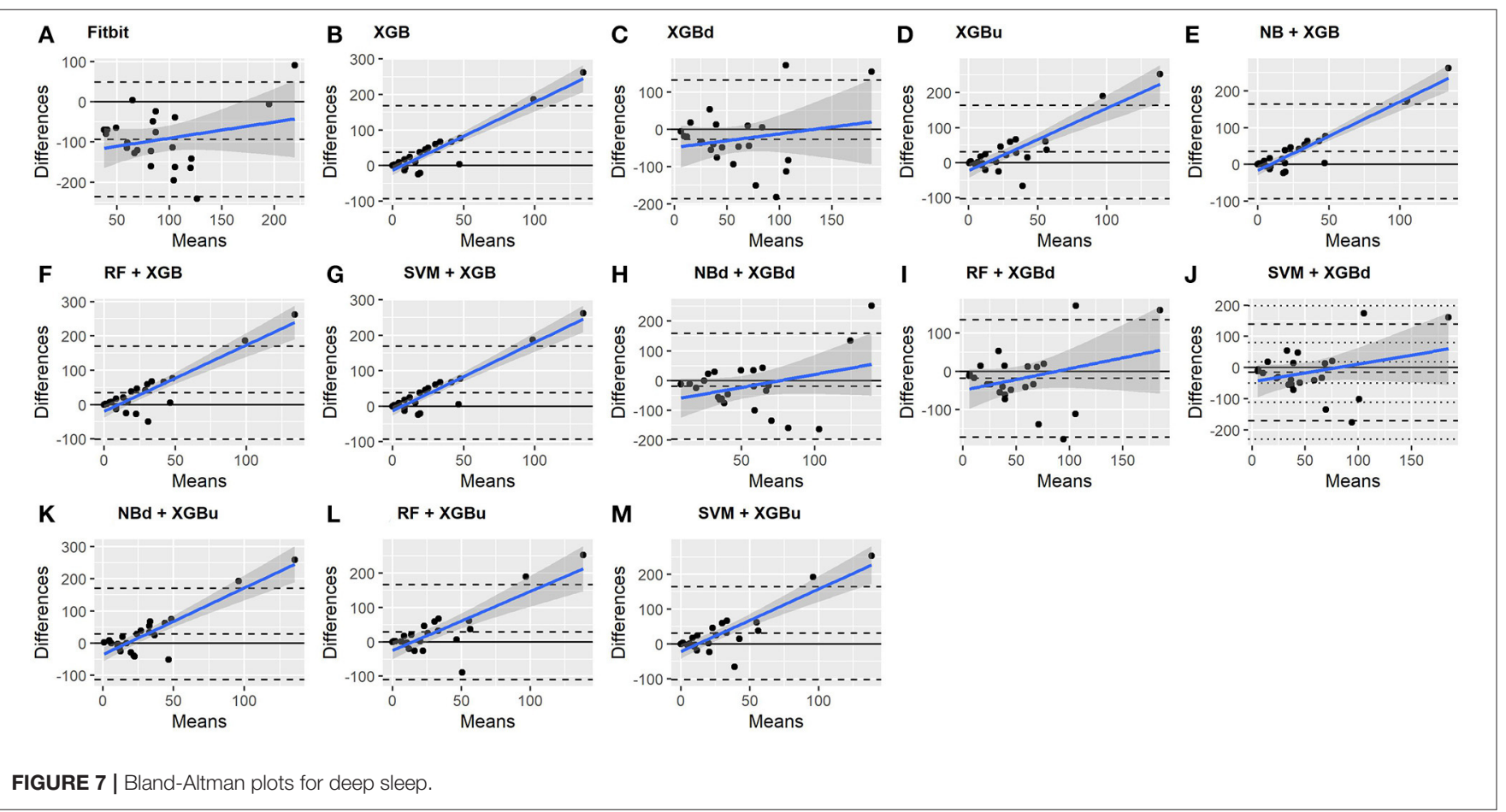

As for REM sleep, RF + XGBd (mean bias: $17.5 \mathrm{~min}$, bias to limit: $105.12 \mathrm{~min} ;=1.567, p=0.238$ ) achieved the smallest bias to the ground truth among all models. SVM + XGBu (mean bias: $22.0 \mathrm{~min}$, bias to limit: $87.3 \mathrm{~min} ; t=2.372, p=0.027$ ) and SVM + XGBd (mean bias: $22.3 \mathrm{~min}$, bias to limit: $95.4 \mathrm{~min} ; t=$ 2.197, $p=0.039$ ) also had better performance than Fitbit (mean bias: $29.1 \mathrm{~min}$, bias to limit: $92.0 \mathrm{~min} ; t=2.971, p=0.007)$. No model showed statistically significant trend in model difference as a function of model mean.

As for wakefulness, RF + XGBd (mean bias: -3.7 min, bias to limit: $117.3 \mathrm{~min} ; t=-0.296, p=0.770$ ) is the only model that achieved better agreement to the ground truth compared 

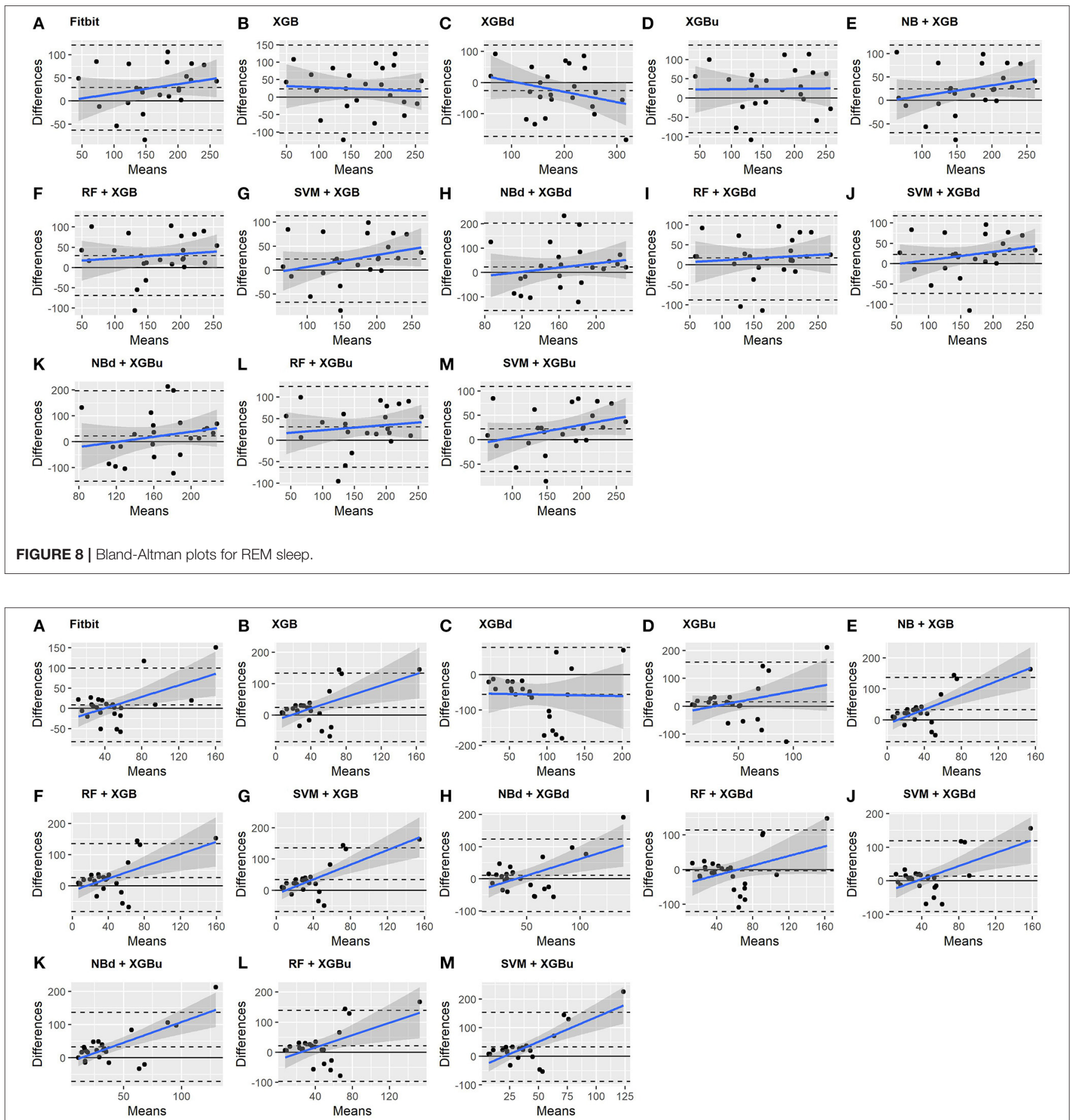

L $\quad \mathrm{RF}+\mathrm{XGBu}$

M $\quad \mathrm{SVM}+\mathrm{XGBu}$
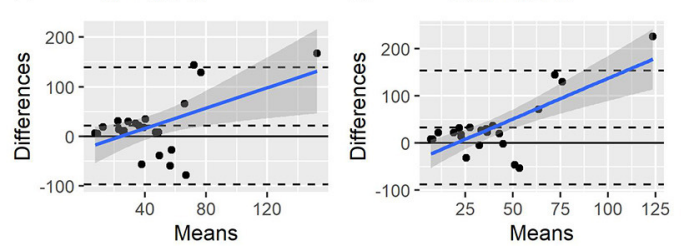

FIGURE 9 | Bland-Altman plots for wakefulness.

to Fitbit (mean bias: 8.7 min, bias to limit: $90.9 ; t=0.894, p=$ 0.381 ). Statistically significant and positive trend is evident along the graph for all models except XGBd, XGBu, and RF + XGBd, and the scatter around the bias line gets larger as the average gets higher for all models.
Figure 10 shows the mean normalized absolute bias (MAB) of all models. RF + XGBd achieved the lowest MAB (0.010), which translates to a $98.4 \%$ decrease compared to that of Fitbit (0.617). All two-stage models achieved lower MAB than Fitbit. Breaking down into the four sleep stages, it shows that the decrease in MAB 


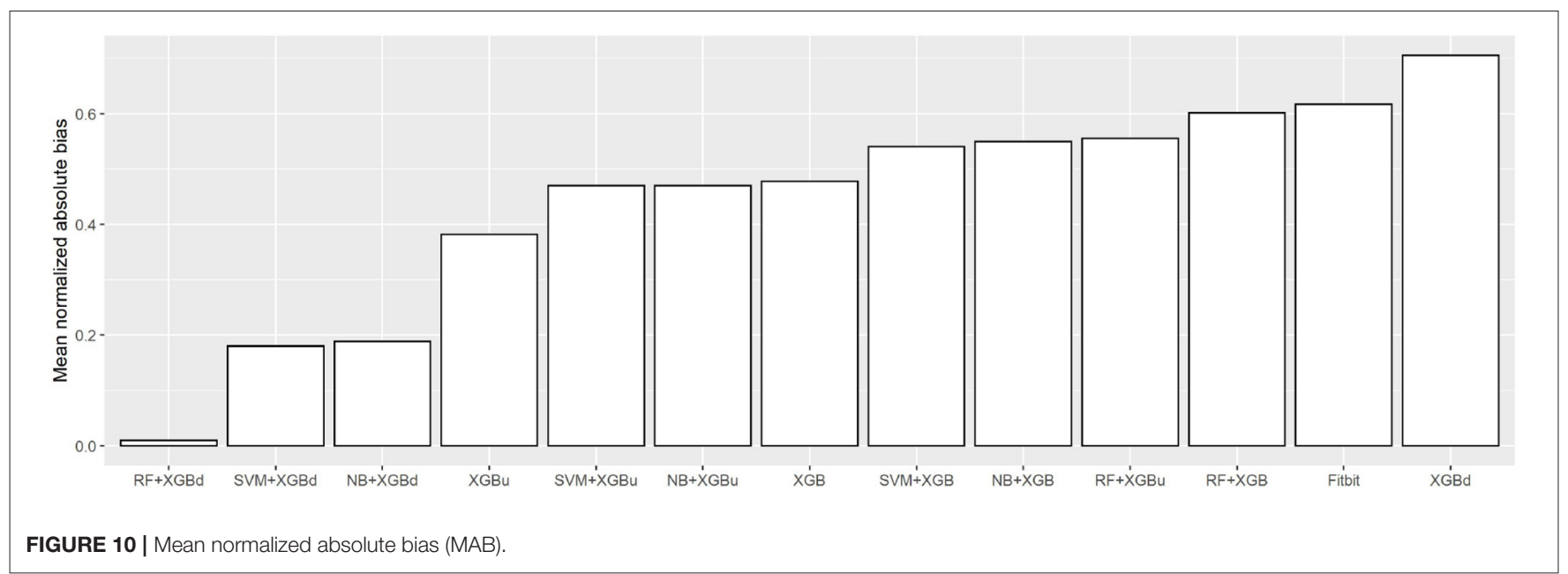

in the two-level models relied on all sleep stages, especially the deep sleep stage.

\section{DISCUSSION}

\subsection{Principal Findings}

This study demonstrates the feasibility of achieving reasonable accuracy in sleep staging with processed data derived from consumer wearables. We have described the evaluation results of the two-level models using multiple performance measures. The two-level models significantly outperform the baseline models in multiple facets when validated against a medicalgrade single channel portable EEG. The best microscopic and macroscopic performance were achieved, respectively by SVM $+\mathrm{XGBu}$ and $\mathrm{RF}+\mathrm{XGBd}$, and SVM + XGBd achieved a good trade off between microscopic and macroscopic performance. Breaking down into individual sleep stage, the best accuracy of the three models was obtained for light sleep (i.e., 89.6\%), whereas prediction accuracy for deep sleep was deteriorated. The two-level models also demonstrated different tendency in misclassification compared to Fitbit. Less light sleep, REM sleep and wake epochs were misclassified as deep sleep, and less REM sleep epochs were misclassified as wakefulness. Nevertheless, more deep sleep and REM sleep epochs were misclassified as light sleep, and more wakefulness epochs were misclassified as REM and light sleep.This indicates that the features constructed using the processed data of the consumer activity tracker may not be sufficient to capture the differences between certain sleep stage pairs.

Regarding overall agreement, the two-level models achieved satisfactory agreement with the ground truth for light sleep and wakefulness. They had lower discrepancy for deep sleep and REM sleep compared to Fitbit, while also eliminating the trends in bias for light sleep and REM sleep. Nevertheless, inconsistent variability was still observed for deep sleep and wakefulness. The scatter around the bias line gets larger as the average gets higher, but such tendency is weaker than Fitbit. Taking all four sleep stages together, the two-level models achieved a MAB of as low as 0.010 , which is $<1 \%$ of the MAB of the baseline models.

We also observed the effect of imbalanced data and dataset shift on model performance. Higher degree of class imbalance of the test set (as quantified by lower value of the Shannon's diversity index) was significantly correlated to deteriorated model performance in multiple dimensions. Resampling at the level-II classification of the two-level models significantly enhanced the overall model performance. While the two-level models using no resampling at level-II performed similarly to or even worse than one level models, both down sampling and up sampling at level-II help improve the model performance with significantly enhanced correcting power. Up sampling generally enhanced microscopic performance, and down sampling allows the models to achieve more balanced accuracy for individual sleep stages and better macroscopic performance. On the other hand, larger dataset shift (as quantified by higher value of the Anderson-darling statistic) was also significantly correlated to deteriorated model performance. The effect was especially manifested in models with up sampling. This is likely due to the side effect of up sampling, which affected the distribution of the three minority classes (while down sampling only affecting one major class) and thus exacerbated dataset shift.

\subsection{Comparison to Prior Work}

While automatic sleep staging with PSG signals has been well-studied for more than two decades, research efforts on approximating sleep stages with widely available consumer activity devices has just started a few years ago.

To our knowledge, there are only three publications attempting four-class sleep stage classification with embedded sensors in consumer activity trackers $(35,50,51)$. Fonseca et al. developed a model using features extracted from ECG heart rate variability and trained with multi-class Bayesian linear discriminant (142 features). This model achieved a Kappa of 0.42 \pm 0.12 and overall per-epoch accuracy of 59\% (51). This model overestimated wakefulness (mean bias $=3.2 \mathrm{~min}$ ), light sleep (mean bias $=28.3 \mathrm{~min}$ ), underestimated REM sleep (mean bias $=41.5 \mathrm{~min}$ ), and agreed well for deep sleep, which yielded a 
mean absolute bias of $18.3 \mathrm{~min}$. Beattie et al. developed models based on raw acceleration and PPG signals from activity trackers using linear discriminant classifiers, quadratic discriminant classifiers, random forest and support vector machine. These models achieved a Cohen's Kappa of up to $0.52 \pm 0.14$ and overall per-epoch accuracy of up to $69 \%$ (50). The model agreed well to the ground truth for deep sleep and wakefulness, whereas underestimated light sleep (mean bias $=11.1 \mathrm{~min}$ ) and overestimated REM sleep (mean bias $=13.0 \mathrm{~min}$ ). The most common misclassifications were light/REM and light/wake mislabeling. This echoes the finding in the present study that the pairs of light/REM and light/wakefulness remains to be challenging to classify based on data from consumer wearable activity trackers. In a recent study, Walch et al. developed sleep staging models using several machine learning algorithms with raw signals from smart watches (35). With artificial neural network, the model achieved the best performance of overall per-epoch accuracy $=72 \%$ and Kappa $=0.30$.

In contrast with the above models that perform sleep classification with raw sensory signals (e.g., acceleration and PPG signals), our approach relies only on processed data from wearable activity trackers (e.g., steps, heart rate, sleep metrics). This strategy sacrifices the granularity of the input data and has to cope with the noise introduced by the proprietary algorithms that are used to process the raw signals, but may increase the feasibility for the models to be implemented in practice, as many wearable devices do not provide public API for retrieving raw sensory signals (15). Our two-level approach adopts a "selective correction" strategy that only reclassifies mislabeled sleep epochs by the manufacturer's proprietary model while keeps labels of the epochs that are correctly classified. The evaluation results demonstrate comparable performance of the two-level models against existing models. That said, there is still much room for improvements especially regarding the accuracy for deep sleep and wakefulness. Possible directions include expanding the feature extraction window, using different set of features at each level of classification, and addressing the class imbalance and dataset shift problems with more advanced techniques.

\subsection{Challenges in Machine Learning Based Sleep Stage Prediction With Consumer Wearable Activity Trackers}

Despite of using only a few manually crafted features, the twolevel models demonstrate promising performance. Enhancing the accuracy of widely used consumer sleep technologies not only brings benefits to the end users by helping them gain a better understanding of their sleep health, but also enables researchers to use these devices to measure sleep outcomes conveniently in longitudinal studies.

As consumer activity trackers take over medical actigraphy with more sensing modalities and functions (52), the opportunities for these devices to be used as a complementary tool in sleep medicine have been increasing $(53,54)$. Nonetheless, there is a need to develop new algorithms, and machine learning is a powerful building block. In the meantime, the present work as well as previous studies highlight several challenges in applying machine learning to sleep stage prediction with consumer devices. Addressing these issues requires inputs from multiple disciplines including sleep medicine, data science, statistics and the consumer wearable industry.

\subsubsection{Challenge 1: Class Imbalance Due to Intrinsic Sleep Stage Distribution in Human Sleep Structure}

Previous sleep studies have shown that the portion of individual sleep stage in a normal night of sleep is highly imbalanced, with light sleep dominating more than $50 \%$ of the total sleep duration (36), which is supported by the sleep data collected in the present study. Applying machine learning algorithms to sleep staging thus face the challenge of class imbalance. Standard machine learning algorithms may bias toward light sleep and yield high learning errors on other sleep stages. Many solutions have been developed to handle the imbalanced problem in the machine learning community. In this study we investigated the effectiveness of two basic resampling techniques. Down sampling at the level-II classification helped achieve more balanced performance for individual sleep stage and significantly reduced systematic bias. Nevertheless, the accuracy for minority classes (especially deep sleep) still has large room for improvement. A possible reason could be that the basic resampling techniques introduced extra noise to the data by affecting the variance of the features, which exacerbated dataset shift and adversely affected classification accuracy. Future studies may explore advanced techniques for handling imbalanced dataset, such as ensemble methods (54) and cost-sensitive learning (55).

\subsubsection{Challenge 2: Dataset Shift Due to Intra/Inter-Individual Variability in Sleep Structure}

Another issue closely related to dataset characteristics is the dataset shift problem which refers to the possible differences in the data distribution for training and test data $(40,56)$. Data shift is a pertinent issue in sleep staging due to dayto-day and inter-individual variability in sleep structure (57, 58). This has been confirmed in the present study using the AD statistic. Dataset shift invalidates the assumption of many supervised learning algorithms that the joint probability distribution remains unchanged between training and testing. It could be a major reason for the high variability of the model performance across participants in this study and in Walch et al. (35). While the issue of dataset shift was not exclusively addressed in this study, future research may employ methods, such as importance-weighted cross validation (IWCV) (59), subclass reestimation (60), GP-based feature extraction (61) and transfer learning (62-64).

\subsubsection{Challenge 3: Lacking a Framework for Model Performance Evaluation}

Machine learning based sleep staging also face the challenge of lacking a framework for model performance evaluation. No consensus has been reached so far as to which measures yield the most objective and unbiased evaluation on sleep staging models. This study evaluates model performance using three measures, which demonstrates slightly different results with varied level of statistical significance. While performance measures, such 
as overall per-epoch accuracy and Cohen's Kappa have been routinely used in previous studies, these measures are not considered as legitimate for imbalanced classification in the machine learning community. Performance measures, such as MMCC adopted in this study may be a promising alternative, but a cut-off value for good performance is yet to be decided. Furthermore, the performance evaluation in this study assumed equal importance of all sleep stages, whereas in practice a certain sleep stage may hold more clinical significance than other sleep stages. For example, the diagnosis of narcolepsy requires the accurate detection of REM sleep. To this end, a universal framework for model performance evaluation should be able to accommodate a wide range of application scenarios.

\subsection{Limitations of Present Study}

The present study has two major limitations. First, a single channel medical EEG device was used as the reference instead of the gold standard PSG. Data recorded using this device may contain noise that negatively affected the supervised model training process. Second, the robustness of the model performance on minor classes-especially deep sleep and wakefulness-demands further improvement. While the macroscopic performance of the two-level models demonstrated good agreement for light sleep, REM sleep and wakefulness, epoch-wise inspection showed that improvement in microscopic performance was limited to light sleep. It was also observed that the model performance heavily relied on the choice of the machine learning techniques and the parameter tuning. These are likely the consequence of the data imbalance and dataset shift issues, which was further exacerbated by the relatively small size of the training dataset. Our further research will focus on addressing these limitations through improving research design

\section{REFERENCES}

1. Tal A, Shinar Z, Shaki D, Codish S, Goldbart A. Validation of contact-free sleep monitoring device with comparison to polysomnography. J Clin Sleep Med. (2017) 13:517-22. doi: 10.5664/jcsm.6514

2. Shin JH, Chee YJ, Jeong DU, Park KS. Nonconstrained sleep monitoring system and algorithms using air-mattress with balancing tube method. IEEE Trans Inform Technol Biomed. (2010) 14:147-56. doi: 10.1109/TITB.2009.2034011

3. Nakamura T, Alqurashi YD, Morrell MJ, Mandic DP. Hearables: automatic overnight sleep monitoring with standardized in-ear EEG sensor. IEEE Trans Biomed Eng. (2020) 67:203-12. doi: 10.1109/TBME.2019.2911423

4. Finan PH, Richards JM, Gamaldo CE, Han D, Leoutsakos JM, Salas R, et al. Validation of a wireless, self-Application, ambulatory electroencephalographic sleep monitoring device in healthy volunteers. J Clin Sleep Med. (2016) 12:1443-51. doi: 10.5664/jcsm.6262

5. Borger JN, Huber R, Ghosh A. Capturing sleep-wake cycles by using dayto-day smartphone touchscreen interactions. NPJ Digit Med. (2019) 2:73. doi: 10.1038/s41746-019-0147-4

6. Ong AA, Gillespie MB. Overview of smartphone applications for sleep analysis. World J Otorhinolaryngol Head Neck Surg. (2016) 2:45-9. doi: 10.1016/j.wjorl.2016.02.001

7. Poyares D, Hirotsu C, Tufik S. Fitness tracker to assess sleep: beyond the market. SLEEP. (2015) 38:1351-2. doi: 10.5665/sleep.4966

8. Choe EK, Consolvo S, Watson NF, Kientz JA. Opportunities for computing technologies to support healthy sleep behaviors. In: Proceedings of the SIGCHI Conference on Human Factors in Computing Systems, CHI'11, ACM Press, New York, NY (2011). doi: 10.1145/1978942.1979395 (e.g., increasing sample size and using PSG data as the ground truth) and applying advanced machine learning techniques (e.g., online machine learning and transfer learning).

\section{DATA AVAILABILITY STATEMENT}

The datasets presented in this study can be found in online repositories. The names of the repository/repositories and accession number(s) can be found at: https://github.com/ PiranitaGomez/Fitbit_sleepStaging_multiLevelML.

\section{ETHICS STATEMENT}

The studies involving human participants were reviewed and approved by School of Engineering, The University of Tokyo. The patients/participants provided their written informed consent to participate in this study.

\section{AUTHOR CONTRIBUTIONS}

ZL conceived and designed the study, performed the data collection experiments, model training and testing, and drafted the paper. MC-M retrieved the Fitbit data, performed the data pre-processing, and made the revision. All authors contributed to the article and approved the submitted version.

\section{FUNDING}

This work was supported by the Japan Society for the Promotion of Science (JSPS) KAKENHI Grant Numbers 16H07469, 19K20141, and 21K17670.

9. Liu W, Ploderer B, Hoang T. In bed with technology: challenges and opportunities for sleep tracking. In: Proceedings of the Annual Meeting of the Australian Special Interest Group for Computer Human Interaction, OzCHI'15, ACM Press, New York, NY (2015). doi: 10.1145/2838739.2838742

10. Ravichandran R, Sien SW, Patel S, Kientz JA, Pina LR. Making sense of sleep sensors: how sleep sensing technologies support and undermine sleep health. In: Proceedings of the 2017 CHI Conference on Human 469 Factors in Computing Systems, CHI'17, ACM Press, New York, NY (2017). doi: 10.1145/3025453.3025557

11. Liang Z, Ploderer B. Sleep tracking in the real world: a qualitative study into barriers for improving sleep. In: Proceedings of the 28th Australian Conference on Computer-Human Interaction, OzCHI'16, ACM Press, New York, NY (2016). doi: 10.1145/3010915.3010988

12. Liang Z, Ploderer B. How does fitbit measure brainwaves: a qualitative study into the credibility of sleep-tracking technologies. Proc ACM Interact Mob Wearable Ubiquitous Technol. (2020) 4:29. doi: 10.1145/3380994

13. Peach HD, Gaultney JF, Ruggiero AR. Direct and indirect associations of sleep knowledge and attitudes with objective and subjective sleep duration and quality via sleep hygiene. J Primary Prev. (2018) 39:555-70. doi: 10.1007/s10935-018-0526-7

14. Weatherall J, Paprocki Y, Meyer TM, Kudel I, Witt EA. Sleep tracking and exercise in patients with type 2 diabetes mellitus (step-D): pilot study to determine correlations between Fitbit data and patient-reported outcomes. JMIR Mhealth Uhealth. (2018) 6:e131. doi: 10.2196/mhealth.8122

15. Liang Z, Ploderer B, Liu W, Nagata Y, Bailey J, Kulik L, et al. SleepExplorer: a visualization tool to make sense of correlations between personal sleep data and contextual factors. Pers Ubiquit Comput. (2016) 20:985-1000. doi: 10.1007/s00779-016-0960-6 
16. Yurkiewicz IR, Simon P, Liedtke M, Dahl G, Dunn T. Effect of Fitbit and iPad wearable technology in health-related quality of life in adolescent and young adult cancer patients. J Adolesc Young Adult Oncol. (2018) 7:579-83. doi: 10.1089/jayao.2018.0022

17. Chang L, Lu J, Wang J, Chen X, Fang D, Tang Z, et al. SleepGuard: capturing rich sleep information sing smartwatch sensing data. Proc ACM Interact Mob Wearable Ubiquitous Technol. (2018) 2:98. doi: 10.1145/3264908

18. Liang Z, Chapa-Martell MA. Validity of consumer activity wristbands and wearable EEG for measuring overall sleep parameters and sleep structure in free-living conditions. J Healthc Inform Res. (2018) 2:152-78. doi: 10.1007/s41666-018-0013-1

19. de Zambotti M, Goldstone A, Claudatos S, Colrain IM, Baker FC. A validation study of Fitbit Charge $2^{\mathrm{TM}}$ compared with polysomnography in adults. $J$ Healthc Inform Res. (2018) 35:465-76. doi: 10.1080/07420528.2017.1413578

20. de Zambotti M, Baker FC, Willoughby AR, Godino JG, Wing D, Patrick $\mathrm{K}$, et al. Measures of sleep and cardiac functioning during sleep using a multi-sensory commercially-available wristband in adolescents. Physiol Behav. (2016) 158:143-9. doi: 10.1016/j.physbeh.2016.03.006

21. de Zambotti M, Baker FC, Colrain IM. Validation of sleep-tracking technology compared with polysomnography in adolescents. Sleep. (2015) 38:1461-8. doi: $10.5665 /$ sleep. 4990

22. de Zambotti M, Claudatos S, Inkelis S, Colrain IM, Baker FC. Evaluation of a consumer fitness-tracking device to assess sleep in adults. Chronobiol Int. (2015) 32:1024-8. doi: 10.3109/07420528.2015.1054395

23. Kang SG, Kang JM, Ko KP, Park SC, Mariani S, Weng J. Validity of a commercial wearable sleep tracker in adult insomnia disorder patients and good sleepers. J Psychosom Res. (2017) 97:38-44. doi: 10.1016/j.jpsychores.2017.03.009

24. Sánchez-Ortuño MM, Edinger JD, Means MK, Almirall D. Home is where sleep is: an ecological approach to test the validity of actigraphy for the assessment of insomnia. J Clin Sleep Med. (2010) 6:21-9. doi: $10.5664 /$ jcsm. 27706

25. Kolla BP, Mansukhani S, Mansukhani MP. Consumer sleep tracking devices: a review of mechanisms, validity and utility. Expert Rev Med Dev. (2016) 13:497-506. doi: 10.1586/17434440.2016.1171708

26. Cook JD, Prairie ML, Plante DT. Utility of the Fitbit Flex to evaluate sleep in major depressive disorder: a comparison against polysomnography and wrist-worn actigraphy. J Affect Disord. (2017) 217:299-305. doi: 10.1016/j.jad.2017.04.030

27. Fokkema T, Kooiman TJM, Krijen WP, van Der Schans CP, de Groot M. Reliability and validity of ten consumer activity trackers. BMC Sports Sci Med Rehabil. (2017) 49:1146. doi: 10.1249/MSS.0000000000001146

28. Liang Z, Chapa-Martell MA. Accuracy of Fitbit wristbands in measuring sleep stage transitions and the effect of user-specific factors. JMIR Mhealth Uhealth. (2019) 7:e13384. doi: 10.2196/13384

29. Liang Z, Martell MAC. Combining numerical and visual approaches in validating sleep data quality of consumer wearable wristbands. In: Proceedings of 2019 IEEE International Conference on Pervasive Computing and Communications Workshops (PerCom Workshops), PerCom'19, Kyoto: IEEE (2019). doi: 10.1109/PERCOMW.2019.8730805

30. Martin JL, Hakim AD. Wrist actigraphy. Chest. (2011) 139:1514-27. doi: $10.1378 /$ chest.10-1872

31. Mitchell TM. (2006). The Discipline of Machine Learning. Machine Learning Department technical report CMU-ML-06-108. Pittsburgh, PA: Carnegie Mellon University. Available online at: http://www.cs.cmu.edu/ tom/pubs/MachineLearning.pdf

32. Buysse DJ, ReynoldsIII CF, Monk TH, Berman SR, Kupfer DJ. The Pittsburgh sleep quality index: a new instrument for psychiatric practice and research. Psychiatry Res. (1989) 28:193-213. doi: 10.1016/0165-1781(89)90047-4

33. McCall C, McCall WV. Objective vs. subjective measurements of sleep in depressed insomniacs: first night effect or reverse first night effect? J Clin Sleep Med. (2012) 8:59-65. doi: 10.5664/jcsm.1664

34. Chambon S, Galtier MN, Arnal PJ, Wainrib G, Gramfort A. A deep learning architecture for temporal sleep stage classification using multivariate and multimodal time series. IEEE Trans Neural Syst Rehabil Eng. (2018) 26:758-69. doi: 10.1109/TNSRE.2018.2813138

35. Walch O, Huang Y, Forger D, Goldstein C. Sleep stage prediction with raw acceleration and photoplethysmography heart rate data derived from a consumer wearable device. Sleep. (2019) 42:zsz180. doi: 10.1093/sleep/ zsz180

36. Ohayon M, Wickwire EM, Hirshkowitz M, Albert SM, Avidan A, Daly FJ, et al. National Sleep Foundation's sleep quality recommendations: first report. Sleep Health. (2017) 3:6-19. doi: 10.1016/j.sleh.2016.11.006

37. Barandela R, Sánchez JS, García V, Rangel E. Strategies for learning in class imbalance problems. Pattern Recogn. (2003) 36:849-51. doi: 10.1016/S0031-3203(02)00257-1

38. Liang Z, Martell MAC. Combining resampling and machine learning to improve sleep-wake detection of Fitbit wristbands. In: Proceedings of the 2019 IEEE International Conference on Healthcare Informatics (ICHI), PICOM'19, Fukuoka: IEEE (2019). doi: 10.1109/ICHI.2019.8904753

39. Bao L, Intille SS. Activity recognition from user-annotated acceleration data. In: Ferscha A, Mattern F, editors. Lecture Notes in Computer Science, Vol. 3001 of PerCom'19. Berlin; Heidelberg: Springer (2004). p. 1-17. doi: 10.1007/978-3-540-24646-6_1

40. Shimodaira H. Improving predictive inference under covariate shift by weighting the log-likelihood function. J Stat Plan Infer. (2000) 90:227-44. doi: 10.1016/S0378-3758(00)00115-4

41. Quionero-Candela J, Sugiyama M, Schwaighofer A, Lawrence ND. Dataset Shift in Machine Learning. Cambridge, MA: The MIT Press (2009). doi: 10.7551/mitpress/9780262170055.001.0001

42. Delgado R, Tibau XA. Why Cohen's Kappa should be avoided as performance measure in classification. PLOS ONE. (2019) 14:e0222916. doi: 10.1371/journal.pone.0222916

43. Chicco D, Jurman G. The advantages of the Matthews correlation coefficient (MCC) over F1 score and accuracy in binary classification evaluation. BMC Genomics. (2020) 21:6. doi: 10.1186/s12864-019-6413-7

44. Fleiss JL, Cohen J. The equivalence of weighted kappa and the intraclass correlation coefficient as measures of reliability. Educ Psychol Meas. (1973) 33:309. doi: 10.1177/001316447303300309

45. Jurman G, Riccadonna S, Furlanello C. A comparison of MCC and CEN error measures in multi-class prediction. PLoS ONE. (2012) 7:e41882. doi: 10.1371/journal.pone.0041882

46. Gorodkin J. Comparing two K-category assignments by a Kcategory correlation coefficient. Comput Biol Chem. (2004) 28:367-74. doi: 10.1016/j.compbiolchem.2004.09.006

47. Fisher RA, Williams ASCCB. The relation between the number of species and the number of individuals in a random sample of an animal population. $J$ Anim Ecol. (1943) 12:42-58. doi: 10.2307/1411

48. Jäntschi L, Bolboacă SD. Computation of probability associated with Anderson-Darling statistic. Mathematics. (2018) 6:88. doi: $10.3390 /$ math6060088

49. Bland J, Altman D. Statistical methods for assessing agreement between two methods of clinical measurement. Lancet. (1986) 327:307-10. doi: 10.1016/S0140-6736(86)90837-8

50. Beattie Z, Oyang Y, Statan A, Ghoreyshi A, Pantelopoulos A, Russell A, et al. Estimation of sleep stages in a healthy adult population from optical plethysmography and accelerometer signals. Physiol Meas. (2017) 38:1968-79. doi: 10.1088/1361-6579/aa9047

51. Fonseca P, Weysen T, Goelema MS, Møst EIS, Radha M, Scheurleer CL, et al. Validation of photoplethysmography-based sleep staging compared with polysomnography in healthy middle-aged adults. Sleep. (2017) 40:zsx097. doi: 10.1093/sleep/zsx097

52. Goldstone A, Baker FC, de Zambotti M. Actigraphy in the digital health revolution: still asleep? Sleep. (2018) 41:zxy120. doi: 10.1093/sleep/zsy120

53. de Zambotti M, Cellini N, Goldstone A, Colrain IM, Baker FC. Wearable sleep technology in clinical and research settings. Appl Sci. (2019) 51:1538-57. doi: 10.1249/MSS.0000000000001947

54. Khoshgoftaar TM, Hulse JV, Napolitano A. Comparing boosting and bagging techniques with noisy and imbalanced data. IEEE Trans Syst Man Cybernet A Syst Hum. (2011) 41:552-68. doi: 10.1109/TSMCA.2010.2084081

55. Iranmehr A, Masnadi-Shirazi H, Vasconcelos N. Cost-sensitive support vector machines. Neurocomputing. (2019) 343:50-64. doi: 10.1016/j.neucom.2018.11.099

56. Moreno-Torres JG, Raeder T, Alaiz-Rodriguez R, Chawla NV, Herrera F. A unifying view on dataset shift in classification. Pattern Recogn. (2012) 45:521-30. doi: 10.1016/j.patcog.2011.06.019 
57. Yetton BD, McDevitt EA, Cellini N, Shelton C, Mednick SC. Quantifying sleep architecture dynamics and individual differences using big data and Bayesian networks. PLoS ONE. (2017) 13:e0194604. doi: 10.1371/journal.pone.0194604

58. Redline S, Kirchner HL, Quan SF, Gottlieb DJ, Kapur V, Newman A. The effects of age, sex, ethnicity, and sleep-disordered breathing on sleep architecture. Arch Intern Med. (2004) 164:406-18. doi: 10.1001/archinte.164.4.406

59. Sugiyama M, Krauledat M, Muller KR. Covariate shift adaptation by importance weighted cross validation. J Mach Learn Res. (2007) 8:985-1005. doi: 10.5555/1314498.1390324

60. Alaiz-Rodriguez R, Guerrero-Curieses A, Cid-Sueiro J. Class and subclass probability re-estimation to adapt a classifier in the presence of concept drift. Neurocomputing. (2011) 74:2614-2623. doi: 10.1016/j.neucom.2011.03.019

61. Moreno-Torres JG, Llorá X, Goldberg DE, Bhargava R. Repairing fractures between data using genetic programming-based feature extraction: a case study in cancer diagnosis. Inform Sci. (2013) 222:805-23. doi: 10.1016/j.ins.2010.09.018

62. Li Q, Li Q, Cakmak A, Poian GD, Bliwise D, Vaccarino V, et al. Transfer learning from ECG to PPG for improved sleep staging from wrist-worn wearables. Physiol Meas. (2021). doi: 10.1088/1361-6579/abf1b0
63. Mehdi Abdollahpour AF Tohid Yousefi Rezaii, Saad I. Transfer learning convolutional neural network for sleep stage classification using two-stage data fusion framework. IEEE Access. (2020) 8:180618-32. doi: 10.1109/ACCESS.2020.3027289

64. Bird JJ, Kobylarz J, Faria DR, Ekart A, Ribeiro EP. Cross-domain MLP and CNN transfer learning for biological signal processing: EEG and EMG. IEEE Access. (2020) 8:54789-801. doi: 10.1109/ACCESS.2020.29 79074

Conflict of Interest: The authors declare that the research was conducted in the absence of any commercial or financial relationships that could be construed as a potential conflict of interest.

Copyright (C) 2021 Liang and Chapa-Martell. This is an open-access article distributed under the terms of the Creative Commons Attribution License (CC BY). The use, distribution or reproduction in other forums is permitted, provided the original author(s) and the copyright owner(s) are credited and that the original publication in this journal is cited, in accordance with accepted academic practice. No use, distribution or reproduction is permitted which does not comply with these terms. 